\title{
El modelo de una agencia de comunicación de moda en España: Tal Cual Comunicación
}

\author{
Recibido: 31 de julio de 2014 \\ Aceptado: 02 de febrero de 2015 \\ Publicado: 30 de octubre de 2015
}

\author{
Ruth Martín Cerro \\ ruthmartincerro@gmail.com
}

Universidad CEU San Pablo (España)

Resumen: Este estudio se enfoca en el sector de la moda en España, a fin de transmitir la necesidad y la importancia de la comunicación en la moda. Esta es una industria que opera en España y posee un plan estratégico de comunicación, llevado a cabo por profesionales dentro del departamento de comunicación interno o bien mediante la contratación externa de una agencia de comunicación especializada en moda. Al analizarse un caso práctico, podemos entender cómo actúa diariamente una agencia en España y cómo lleva su plan estratégico de comunicación, el cual resulta vital, ya que responde a una necesidad de consultoría o asesoramiento personalizado de una agencia de comunicación hacia sus clientes.

Palabras claves: Agencia de comunicación, comunicación empresarial, sector de moda, España.

\begin{abstract}
The purpose of this paper is to research how Spanish fashion works. A case study of a Spanish fashion communication agency: Tal Cual Comunicación is meant to show the necessity and the importance of communication in fashion. Fashion is one of industries in Spain and needs to have a strategic plan of communication, which would be led by professional people who work either at a communication department or communication agency specialist in fashion. We can understand through this study case how this industry works and deal with a communication agency by working on a communication strategy plan. To sum up, we can see that both the objectives and the hypothesis match with the conclusions. This means that there is a necessity of a personal treatment from a communication agency to its clients.
\end{abstract}

Key words: Communication Agency, Business Corporate Communication, Fashion Industry, Spain. 


\section{Introducción}

Con el fin de conocer cómo se estructura y funciona una agencia de comunicación de moda es necesario investigar en profundidad y comprender el concepto de la comunicación empresarial e institucional actual. Apoyándonos en el modelo español de Tal Cual Comunicación, podremos averiguar cómo interactúa con las firmas que confían en su labor y los medios de comunicación españoles. Para poder llegar a este estudio, se explica, en primer lugar, qué se entiende por una agencia de comunicación y se repasan las diferentes formas de comunicar la moda, sea a través de instituciones, empresas, agencias de comunicación y formación en la comunicación de moda.

Con la finalidad de alcanzar la objetividad y pluralidad, se realizaron entrevistas a profesionales de este sector: al modisto Lorenzo Caprile; a la directora de Sector de la Agencia de Comunicación Equipo Singular, Vanesa Peloche; al consultor de Comunicación especializado en los sectores del lujo y de la moda, Ignacio Espada; a la directora de Comunicación de la agencia de comunicación Tal Cual Comunicación, Paula Gullón; a la bloggera y periodista de moda, Fátima Fontcuberta.

Nuestra investigación, descriptiva y analítica, la hemos basado en el ámbito nacional español, específicamente en la agencia Tal Cual Comunicación. El proceso de elaboración del trabajo está dividido en dos etapas:

a) Investigación básica o teórica:

- Búsqueda y análisis de fuentes bibliográficas, hemerográficas y digitales.

- Uso de diccionarios específicos dentro del sector que nos ocupa.

- Investigación y creación, mediante el análisis de las diversas fuentes y de los puntos clave del trabajo.

b) Investigación aplicada o praxis:

- Acceso a la información cualitativa y cuantitativa de la propia agencia de comunicación, a través de su último informe anual o memoria y resto de datos desarrollados anualmente por la misma.

- Contacto con especialistas en el ámbito de la comunicación empresarial e institucional de la moda, en concreto con sus direcciones de comunicación.

- Conclusiones finales.

\section{Análisis del mundo de la moda}

\subsection{Conceptualización de moda}

De acuerdo con la Real Academia Española (2012a: 733-734), la moda "es un uso, modo o costumbre que está en boga durante algún tiempo, o en determinado país, con especialidad en los trajes, telas y adornos, principalmente los recién introducidos". Para la Asociación de Creadores de Moda de España (2011), la moda es concebida "como cultura y como 
economía, por lo que su objetivo es fomentar y desarrollar actividades que redunden en beneficio del sector del diseño de moda en sus vertientes creativa y comercial, realizando un constante estudio y seguimiento de los mercados nacionales e internacionales y participando en misiones comerciales de cualquier índole, especialmente en países estratégicos”.

Según Laver (2006: 10-12), "la historia del traje comienza mucho antes de que las primeras civilizaciones de Egipto y Mesopotamia hicieran su aparición. En los últimos años, un gran número de descubrimientos y el estudio de las pinturas rupestres han proporcionado documentación mucho más antigua. Para Deslandres (1998: 17-18) el traje, nos guste o no, "forma parte incuestionable del confort humano. Los filósofos que se han interesado por el fenómeno, siempre han insistido en su significación social. Aceptar vestirse es formar parte de la sociedad civilizada".

Hoy en día, muchos diseñadores se valen de sus colecciones de ropa, ya sean de alta costura o de prêt-à-porter, como forma de conseguir notoriedad, lo que a su vez vende producto. Los diseñadores crean una marca o imagen mediante innovaciones de estilo en sus colecciones pero, en realidad, el beneficio lo obtienen más de las licencias que de las colecciones de pasarela. Si el nombre del artista no tiene una jerarquía de marca, las licencias no tienen valor. En un acuerdo de mercadotecnia (conjunto de técnicas, estrategias y aplicaciones para la consecución de los objetivos de marketing) que recorra todo el proceso, las licencias financian las colecciones que, a su vez, generan expectativa suficiente para la venta de las licencias. Esta práctica empresarial permite que los diseñadores amplíen las fronteras de la innovación y marquen el futuro de la moda ( $c f$. Palomo-Lovinski, 2011: 8-9).

\subsection{El sector de la moda en España}

Actualmente, los dos cimientos en los que se asienta la moda en nuestro país son el sector público y el sector privado.

\subsubsection{Sector público}

Las financiaciones públicas en España destinadas al mundo de la moda recaen en las siguientes organizaciones:

- Semana Internacional de la Moda de Madrid, organizada por IFEMA, la Institución Ferial de la capital. Entre sus diferentes actividades y exposiciones tiene cabida la Mercedes-Benz Fashion Week Madrid, de frecuencia bianual y principal plataforma para la promoción de la moda en España. Los diseñadores nacionales más influyentes son Ágatha Ruiz de la Prada, Amaya Arzuaga, Ana Locking y Devota \& Lomba. Además, los diseñadores noveles tienen un espacio en este evento tan importante bajo el nombre de EGO. Este nació en febrero 2006, con el fin de dar a conocer nuevos diseñadores que entendieran la moda como una forma de expresión, como una disciplina artística que forma parte de la cultura de su tiempo, con la justificación final de llegar a la calle, ser aceptada por la sociedad y continuar así el camino de la evolución descubriendo nuevos estilos y tendencias innovadoras. 
- La 080 Barcelona Fashion o Semana de la Moda de Barcelona es la pasarela que acoge la Ciudad Condal con los objetivos de convertir a Cataluña en el referente de creatividad, innovación, generación y proyección del diseño en el sector de la moda. Entre sus diseñadores se encuentran la firma Mango, TCN y Desigual. Se celebra también la Barcelona Bridal Week Novia España de carácter anual, que se centra en el Salón Profesional de la Moda en Vestidos de Novia, Novio, Fiesta, Comunión, Ceremonia y Complementos. Además, se encuentra la Pasarela Gaudí Novias, también anual.

- El Instituto Español de Comercio Exterior (ICEX) es una entidad pública empresarial de ámbito nacional que tiene como misión promover la internacionalización de las empresas españolas para contribuir a su competitividad y aportar valor a la economía en su conjunto, entre ellas las de moda.

- El Museo del Traje, Centro de Investigación del Patrimonio Etnológico, situado en el centro de Madrid, pertenece a la red de museos estatales y está coordinado por el Ministerio de Cultura. Desde su creación (2004) posee y alberga en sus instalaciones una amplia colección y un gran fondo etnológico debido a su evolución histórica.

\subsubsection{Sector privado}

Este quedaría dividido en dos partes: las asociaciones profesionales y las diversas empresas de moda.

- En España existen multitud de asociaciones relacionadas con el mundo de la moda que se crean con el fin de defender, difundir y promocionar los valores de la moda nacional, así como de un determinado sector especializado de este. Algunas de ellas son: ASSOMODA, la Federación de Industrias del Calzado Español (FICE), la Federación Española de Empresas de la Confección (FEDECON), la Agrupación Española del Género de Punto (AEGP) y la Asociación Española de Joyeros, Plateros y Relojeros (AEJPR). Sin embargo, cabe destacar dos de estas: la Asociación de Creadores de Moda de España (ACME) y la Asociación Artística de Diseñadores Nacionales de Moda de España (ADÑ). La primera de ambas es una organización profesional sin ánimo de lucro integrada por diseñadores de todo el país. Con sede en Madrid capital, ACME es fundada en 1998 y en la actualidad tiene asociadas 38 marcas de diseño de moda. Entre sus principales objetivos se encuentran el estudio y la difusión de todo tipo de medidas encaminadas a promocionar la imagen exterior de la moda española. Destacan entre sus asociados David Delfín, Amaya Arzuaga, Kina Fernández, Andrés Sardá, y Ágatha Ruiz de la Prada, todos ellos presididos por el diseñador gallego Modesto Lomba. La Asociación Artística de Diseñadores Nacionales de Moda de España fue presentada en la edición del año 2007 de la Semana Internacional de la Moda de Madrid y responde a la necesidad de crear una asociación de jóvenes diseñadores, independiente de la popular ACME, con un objetivo claro: fomentar una imagen de la moda de España. El Ministerio de Asuntos Exteriores lo ha incorporado dentro de la marca imagen de España. 
- Actualmente, las existentes empresas de moda están, en su mayoría, englobadas en las citadas asociaciones profesionales. Haciendo un total, entre nacionales e internacionales, de aproximadamente 608 ( $c f$. Espada, 2015).

Agatha Ruiz de la Prada cree en el made in Spain: "Hacer la guerra por tu cuenta es muy negativo. Tienes que hacer la guerra en batallones. Creo que el ir juntos, tener algo que decir distinto respecto a los demás, eso ayuda. Lo que pasa es que es difícil. Hay algo común entre los españoles y hay que hacerlo valer [...] Ahora, en España, tenemos la cocina y la arquitectura, que están en un momento óptimo. Habría que salir con eso junto a la moda. Pero ahí hace falta un líder político que lo organice con buena comunicación, y me parece difícil. Hay quien dice que lo que se lleva son las ciudades: Milán, París, etc. Pues no existe ni el 'made in París' ni el 'made in Milán'. Existen la moda francesa y la moda italiana. No creo en el 'made in Madrid' ni el 'made in Costa Brava', me parece una tontería” (ápud Massot, 2008: 188-189).

Por otra parte, es cierto que los diseñadores se encuentran con problemas. El modisto español Juan Duyos lo explica: "En España es un delirio lo que ocurre en el mundo de la moda, en ningún país un diseñador está solo; tiene un grupo empresarial detrás, que le defiende y apoya y hacen dinero con su idea. Aquí no existe, y mientras no exista ese modelo, la moda española no va a funcionar" (Chávez, 2009: 462-470).

\subsection{Empresas de moda}

El sector textil y de la confección ocupa un lugar importante dentro de la estructura industrial española, ya que desde el 2008 brinda trabajo directo a más de 200 mil personas, cifra que representa el $7 \%$ del empleo industrial. Su aportación al producto industrial es del 4\% del total (Comunidad de Madrid, 2009). Actualmente, las existentes empresas de moda están, en su mayoría, englobadas en las citadas asociaciones profesionales. Haciendo un total, entre nacionales e internacionales, de aproximadamente 608 ( $c f$. Espada, 2015).

Desde el 2008, la crisis ha borrado del mapa a 300 mil pymes de todos los sectores y ha obligado a las grandes a modificar sus estrategias, como la iniciativa anunciada por el presidente de Mango, Isak Andic, para rebajar toda la nueva colección un 20\% para animar el consumo. De acuerdo con Pastor (2012: 56-60), España atraviesa una "economía de la experiencia y el pequeño comercio tiene más capacidad para crear vivencias únicas frente a las grandes cadenas".

El Instituto Nacional de Estadística (INE) estima que la demanda interna se redujo al 1.1\% en 2011, mientras que los datos de la Asociación Española del Lujo reflejaron un repunte de este sector del $20 \%$ en el mismo período. El bajo coste sigue su crecimiento imparable, con un alza del $30 \%$ en solo cuatro años, según la consultora de alta dirección internacional PriceWaterhouseCoopers y el avance de los outlets virtuales, (plataformas que ofrecen artículos de primeras marcas de anteriores temporadas con descuentos), que ya acaparan el $61 \%$ del mercado online (cf. Actualidad Económica, 2012). 


\subsection{Medios de comunicación especializados}

Los medios de comunicación especializados en moda son muchos y muy variados:

- Prensa: existen prestigiosas revistas de moda en España, tales como Vogue, Elle, Telva, Marie Claire y Neo2, entre otras. También son muy populares los suplementos de moda de grupos editoriales entre los que destacan Yo Dona (Unidad Editorial) y S Moda (PRISA).

- Televisión: los programas de televisión especializados en moda son muy escasos en España. Prácticamente todos son versiones de otros países adaptados a nuestro país. En todo caso, algunas cadenas televisivas dedican pequeños espacios de moda en sus programas de información generalista: "Cosmopolitan TV" (Hearst Entertainment \& Syndication), "Fashion TV" (Turner Broadcasting System) y "Moda con firma" (Decasa).

- Internet: este medio de comunicación es el más sobresaliente en espacios dedicados a la moda gracias a su diversificación de públicos. Destacan las versiones online de las revistas de moda, las asociaciones e instituciones, programas de televisión y medios específicos de moda. El Instituto de Comercio Exterior (ICEX) posee un canal especializado en moda. Fashion from Spain proporciona información sobre distintos subsectores del sector moda, directorio de empresas, noticias, ferias y boletines. Modaes.es ofrece información económica del negocio de la moda en España, la referencia de actualidad para los profesionales, directivos y demás líderes de opinión del sector gracias a su especialización y a su rigor periodístico. Además tiene su propia revista trimestral que da una mayor extensión, profundidad y análisis.

- Blogs: son una plataforma que no se asientan en un lugar concreto. Hay partidarios que afirman que son medios de comunicación y otros que se oponen.

\subsection{Formación en la comunicación de moda}

En España existen posgraduaciones o maestrías dedicadas a la comunicación especializada en la moda y la belleza. La Universidad Carlos III de Madrid desarrolla un programa en colaboración con la revista Vogue. El Centro Universitario Villanueva, adscrito a la Universidad Complutense de Madrid, lanzó el título en "Comunicación y Gestión de la Moda". Asimismo, existe un programa MBA en "Empresas de Moda" en el Instituto Superior de Empresas de Moda (ISEM), pero está centrado en la gestión de empresas de moda, lo mismo que el programa "Dirección y Gestión de Empresas de Moda", del Instituto Europeo de Diseño. Han surgido programas relacionados con la industria y la creación y diseño de moda en universidades catalanas, así como en institutos y centros de formación privados que no tienen el rango universitario. 


\section{Tal Cual Comunicación como agencia de comunicación de moda}

\subsection{Breve historia}

Tal Cual Comunicación nace en enero de 2011 con dos sedes conjuntas: Madrid y Barcelona. La agencia la forman tres socias: Helena Ramada, Marta Nebot y Paula Gullón, quienes habían laborado juntas en otra agencia. Las dos primeras se encuentran en la ciudad condal y la última en la capital. Su principal objetivo fue crear un proyecto propio donde la comunicación sea la herramienta principal, llegando a formar parte de los clientes. $\mathrm{Su}$ finalidad fue tender un puente entre la marca de moda y la prensa española y, para ello, se deben conocer muy bien las necesidades de ambas partes. Por ello, el diálogo resultó fundamental para tener claros los objetivos de los clientes.

La agencia se define de la siguiente manera: "Tal Cual es sinónimo de equipo, trabajo, dedicación y amor por la moda en todos sus sentidos. Somos una agencia joven que nace con la ilusión de unas principiantes y la experiencia de unas veteranas. Nos gusta involucrarnos, conocer y cuidar a nuestros clientes. Nos alejamos de los personalismos y apostamos por la gente que se apasiona con sus proyectos. La comunicación constante, fluida y natural mueve nuestro mundo. Así somos, tal cual”.

\subsection{Estructura y organigrama}

Una agencia de comunicación debe tener una estructura de al menos seis departamentos imprescindibles, como son: la dirección general, la dirección de comunicación, contactocuentas, creatividad, investigación, planificación estratégica y financiación ( $c f$. Martín Martín, 2010: 171-193). El actual organigrama de Tal Cual Comunicación (figura 1) es el siguiente:

Figura 1: Mapa contextual para el desarrollo del periodismo de datos.

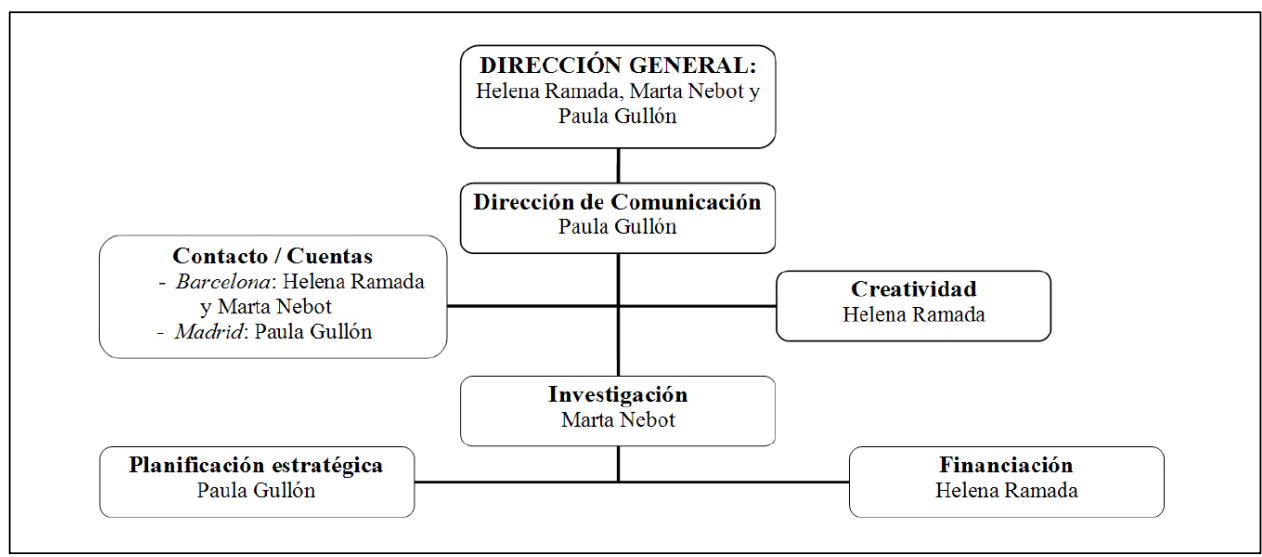

Fuente: Martín Cerro (2010). 
- Dirección General: corre a cargo de las tres socias conjuntamente. Al tener dos sedes, Paula Gullón se encarga de poner al día la delegación de Madrid, mientras que Helena Ramada y Marta Nebot lo hacen en Barcelona. Se comunican diariamente por teléfono y una vez al mes tienen una reunión de trabajo para supervisar todas las acciones realizadas.

- Dirección de Comunicación: emprende la gestión de la comunicación interna y externa, así como de la imagen de la agencia. Este departamento es el pilar fundamental de la empresa, que la lleva a cabo Paula Gullón desde Madrid. Siempre en comunicación permanente con sus otras dos socias, quienes también son partícipes en todo momento de lo que conlleva esta labor.

- Contacto-cuentas: cada firma la lleva personalmente cada una de las socias, quienes están en contacto permanente con el cliente y los medios de comunicación. Tanto Gullón, Ramada y Nebot ejecutan las notas de prensa o informativas; clippings o seguimiento de medios, ruedas de prensa y dosieres especializados, entre otros, de cada una de las marcas que tienen a su cargo.

- Investigación: Nebot es la que desarrolla todos los estudios y análisis, siempre en coordinación con las otras dos socias.

- Creatividad: las tres socias son las que piensan y desarrollan cada acción y después Ramada es la que da forma a las ideas y las diseña.

- Planificación estratégica: al tener la responsabilidad de llevar la Dirección de Comunicación, Gullón es la que tiene un trato más directo y confiado con los medios para desarrollar todas las acciones, ya sean informativas o publicitarias.

- Financiación: Ramada se encarga de llevar al día estas funciones financieras.

\subsection{Funciones del Departamento de Comunicación}

\subsubsection{Análisis de los públicos de interés}

- Clientes o retail: son las empresas especializadas en la comercialización masiva de productos o servicios uniformes a grandes cantidades de clientes. Al igual que existen clientes que llevan una sola firma, los llamados retail se encargan de la comercialización y distribución de varias marcas y son estos los que contratan directamente a la agencia. Ejemplos de estos clientes en Tal Cual Comunicación son la plataforma de Internet Yigibee y QB Distribuciones.

- Prescriptores de la marca: bloggers y celebrities en su mayoría.

- Bloggers: en su mayoría son profesionales jóvenes que escriben sobre el street style, aconsejando a los lectores sobre las distintas tendencias de las firmas. 
- Celebrities: a través de estas, la marca logra un buen posicionamiento en el mercado de la moda. Las agencias regalan varios productos de las firmas a personas de relevancia mediática para que puedan ser fotografiadas con la prenda o accesorio y salir en revistas de moda. El objetivo es el mismo: dar a conocer la marca al futuro cliente.

- Medios de comunicación: mediante una base de datos contrastada, el Dircom es el encargado en ponerse en contacto con dichos medios para posicionar sus firmas de moda en el mercado y que halla una constante comunicación entre ellos y los clientes.

- Público final o compradores: es uno de los objetivos de las firmas al contratar los servicios de una agencia de comunicación. Al tener sus marcas en un buen posicionamiento, el futuro comprador conoce mejor la firma y se determina su futura decisión de compra.

\subsubsection{Política comunicativa y plan estratégico de comunicación}

- Qué: análisis de la situación del sector en el mercado a estudiar. En el caso de Tal Cual Comunicación, el mercado objetivo es la moda y para estudiarlo las tres socias han hecho una investigación exhaustiva viendo las fortalezas y debilidades, así como las oportunidades y amenazas de este sector para hacerse un espacio en él.

- Quién: investigar personalizadamente y en equipo las necesidades comunicativas del cliente, a través de una previa auditoría de comunicación. El primer objetivo al crear la agencia era encontrar clientes que creyesen en su política. Para ello, las tres socias (con datos concretos) tuvieron que hacer una presentación profesional personalizada para intentar captarlos. Una vez que ya formaron parte del equipo, lo más importante y delicado fue seguir la misma línea de actuación para que esa confianza y profesionalidad no cese. Por ello, la buena y directa comunicación entre la agencia y los clientes es vital e imprescindible.

- Cómo: realización del plan o estrategia basado en información, publicity (todo tipo de información de interés público que emite una agencia hacia sus públicos de interés de forma gratuita), relaciones públicas e imagen (notas informativas, videonoticias, elaboración de clippings, shopping (muestra de todas las prendas y complementos de los clientes para que las celebrities, estilistas y la prensa puedan escoger para su posterior utilización), eventos, ruedas de prensa, participación en ferias de moda como Little Barcelona, informe anual o memoria, manual de identidad/imagen, portal web y vinculación a redes sociales especializados.

- Cuándo: el cliente va definiendo sus necesidades, según observa la profesionalidad y calidad de las acciones comunicativas de Tal Cual Comunicación, tomando la confianza mutua que es necesaria en este tipo de actividad de confidencialidad. 
-Dónde: según esas necesidades concretas, la agencia va proponiendo y apareciendo en los medios oportunos, siempre previa consulta al cliente, tanto a nivel informativo, relaciones públicas, publicity o publicitariamente.

- Por qué: una vez cumplidas estas necesidades, se observa si los servicios de la agencia han sido rentables al Dircom, consiguiendo una eficaz fidelización del mismo.

Según Paula Gullón, el plan estratégico de comunicación "depende de las necesidades de cada marca. Para cada una de ellas se elabora un plan estratégico con el fin de sacar el mayor rendimiento posible a nuestros servicios, teniendo en cuenta su tipología, a quién quieren llegar, qué quieren transmitir [...] Desde una presentación en exclusiva a la que se convoca a todos los medios, hasta un viaje patrocinado por alguna de las marcas [...] Aunque en estos tiempos, las marcas piden el máximo rendimiento al menor coste y hay que saber ingeniárselas para hacer acciones impactantes a bajo coste".

\subsubsection{Acciones de comunicación interna}

Entre sus muchas funciones, la agencia elaboró en su comienzo un organigrama detallando cada una de las labores de los departamentos. Acordando que diariamente harían una videoconferencia o, en su defecto, una llamada de grupo, puesto que tienen dos sedes. Con esto consiguen estar informadas las tres socias de todo cuanto acontece en cada momento. Otra de las acciones de comunicación interna es el análisis diario de medios de comunicación: periódicos generalistas, suplementos, revistas especializadas, televisiones, productoras, webs y blogs. De esta manera se hace un clipping o seguimiento de medios exhaustivo, que ayuda a posicionar las noticias más significativas que afecten al sector y a la agencia.

Tal Cual cuenta, como ya hemos mencionado, con un amplio banco de datos con diversas bases de datos, que van aumentando cada día, debido al boom que se ha creado con los blogs de moda. En dichas bases de datos no solo se encuentran los contactos de los clientes o medios más cercanos que tienen un trato ya casi diario, sino los posibles futuros clientes y todos los medios que se van haciendo un hueco en el periodismo.

Se elaboran grupos focales o reunión de grupos de opinión-discusión sobre marcas, en los que con participación de públicos con unas variables determinadas, se detectan las bases para futuras acciones comunicativas sobre la moda. Sobre el mes de enero de cada año (una vez presentados y aprobados los presupuestos generales de la agencia) se realiza un informe y resumen de las actividades sociales y económicas realizadas por cada una de las áreas y departamentos. De esta forma, se estructura la memoria anual.

\subsubsection{Acciones de comunicación externa}

La Agencia redacta y elabora los contenidos que puedan generar el interés de la prensa, webs y blogs especializados, que permitan una presencia permanente de la marca 
en los medios de comunicación tradicionales y online. También elaboran dosieres especializados o carpetas de prensa específicas de cada marca con contenido gráfico y una nota informativa explicando cada colección para enviarlas a los medios de comunicación.

Una de las acciones más importantes es la comunicación. Por este motivo, la agencia siempre está en constante diálogo con los redactores y estilistas gracias al previo banco de datos. Periódicamente se envían newsletters o boletines diarios digitales con noticias puntuales para generar un goteo continuo de información sobre los clientes presentes. Además, se procura contactar diariamente con las firmas para exista una transparencia en cada una de las acciones. La agencia aporta diariamente contenidos a los perfiles de los clientes en todas las redes sociales existentes. De esta manera, la persona encargada pone al día las cuentas de Facebook, Tuenti, Twitter, LinkedIn y Sing, entre otras.

La agencia dispone de showrooms en Madrid y en Barcelona, ambos emplazados en la ruta de shopping de las estilistas. Todas las colecciones de los clientes disponen de un espacio personalizado en las oficinas. De esta manera, las estilistas de los programas de televisión, series, películas y prensa escrita pueden acudir y seleccionar modelos para sus reportajes y bazares. El clipping o seguimiento de medios también es eficaz en la comunicación externa. Así, cada vez que la firma aparezca en un medio de comunicación, se va elaborando un dossier especializado o press-book, donde se incluye la valoración económica de las mismas y se da al cliente cuando lo solicite.

Actualmente, invertir en publicidad supone un esfuerzo y es muy importante rentabilizar al máximo la inversión. Por eso, Tal Cual Comunicación orienta a los clientes sobre qué medios son más afines a su marca y público objetivo. Asimismo, se encargan de la planificación y compra de medios, trabajando en equipo con los responsables de publicidad dentro del departamento de comunicación o cliente para, entre todos, lograr grandes resultados. Otra de las acciones comunicativas es la realización de actos o eventos para crear vínculos entre las marcas y la prensa. Entre estos se proyectan las presentaciones de productos, actividades de carácter lúdico, open day o jornada de puertas abiertas, presentaciones en exclusiva o ventas especiales.

\subsubsection{Responsabilidad social empresarial o corporativa}

Tal Cual Comunicación realiza varias labores de Responsabilidad Social. Entre ellas, su altruismo hacia los trabajos realizados a varias firmas de moda. Y quizá, la más importante, colabora con la marca Toms Shoes en su labor de regalar un par de zapatos nuevos a niños necesitados por cada par vendido, en colaboración con organizaciones no gubernamentales. Con este acto, que supera las cinco ediciones, congregó a celebrities y medios españoles en la terraza del Mercado de San Antón de Madrid.

\subsubsection{Necesidad de las nuevas tecnologías: Comunicación 2.0}

Espada (2015) apunta las claves para que una agencia tenga una buena comunicación 2.0: 
"En el mundo empresarial ni existen formulas mágicas ni medidas que se adecuen a cada firma con éxito. Por lo tanto, hay que elaborar un estudio adecuado sobre nuestra comunicación online, aquella que necesitamos, la que nos va a beneficiar y cómo va a afectar a nuestra arquitectura de marca. Estoy hablando de implementar un plan con unos objetivos concretos y reales. Los departamentos de comunicación de las firmas han de profundizar midiendo y evaluando los medios de comunicación 2.0, comprender y aplicar el posicionamiento SEO (optimización para motores de búsqueda) y SEM (marketing en buscadores web) y coordinarse con marketing. Esto implica inversión, apuesta, experimentación y aplicación práctica con el apoyo de profesionales especializados dentro de la empresa o externos a la estructura empresarial".

Tal Cual Comunicación gestiona intangibles que son muy difíciles de medir. Desarrolla esta modalidad con plataformas de comunicación y soportes técnicos como las redes sociales (Facebook, Tuenti, Twitter, YouTube) y su propio blog. Las tres socias se encargan personalmente de utilizar estos nuevos medios que les permiten una mayor eficacia y un plus de calidad. La finalidad es coordinar estos procesos comunicativos que tienen un impacto directo sobre la optimización de procedimientos, el ahorro de costes y la generación de negocio.

\section{Reflexiones finales}

La moda es un sector muy amplio y en pleno auge que necesita la comunicación para su desarrollo. Al igual que otras industrias, precisa de profesionales que la lleven a cabo. En este caso, como hemos visto, la moda es comunicada a través de instituciones, empresas, medios de comunicación, academias formadoras y especializadas agencias de comunicación.

Una empresa de moda puede llevar su comunicación a través de un departamento de comunicación interno o bien mediante la contratación específica de una agencia de comunicación especializada. Esta última modalidad es la más establecida en España debido a su pluralidad y profesionalidad, sobre todo, en el apoyo y desarrollo de actos o eventos de moda. Para llevar a cabo una buena comunicación es fundamental conocer el sector, el producto y el público al que dirigirse.

\section{Fuentes consultadas}

Actualidad Económica (2012, abril 1). "Las costuras que tejen el éxito”. Núm. 2718, pp. 11-12. Extraída el 21/VII/2015 desde http://www.luxuryspain.es/2012/04/las-costurasque-tejen-el-exito/

Agencia Nacional de Evaluación de la Calidad y Acreditación (2006). "Libro Blanco. 
Títulos de grado en Comunicación”. Extraída el 21/VII/2015 desde http://www.aneca.es/ var/media/ 150336/libroblanco_comunicacion_def.pdf

Asociación Creadores de Moda de España, ACME (2011). "Memoria 2010”. Extraída el 21/VII/2015 desde http://creadores.org/wp-content/uploads/2014/02/memoria2010.pdf

Chávez, B. (2009). El libro rojo del estilo. Madrid: Martínez Roca.

Comunidad de Madrid (2009). "Madrid Emprende. Agencia de Desarrollo Económico. Informe anual 2008". Extraída el 21/VII/2015 desde http://www.madridemprende.es/ Intranet/images_load/ME_2008\%281\%29.pdf

Deslandres, Y. (1998). El traje, imagen del hombre. Barcelona: Tusquets.

DIRCOM (2014). Anuario de la Comunicación. Madrid: Asociación DIRCOM.

East Wind Marketing y Comunicación (2012, febrero 2). "mercedesbenzfashionweekmadrid". Extraída el 21/VII/2015 desde http://www.eastwind.es/blogs/eastwindmarketingycomunicacion/ 2012/02/02/garcia-duran-sustituye-a-irisarri-en-la-presidencia-ejecutiva-de-vertice-360\% $2 \% \mathrm{~B} \mathrm{~A} /$ mercedesbenzfashionweekmadrid/ Espada, I. (2015). Blog Modaes Latinoamérica. Extraída el 21/VII/2015 desde http://www. modaes.co/

Generalitat de Catalunya (2015). “080 Barcelona Fashion”. Extraída el 21/VII/2015 desde http://gener2012.080barcelonafashion.cat/es/bcn080

Kissinger-Matray, D. (1990). "La sponsorización y el mecenazgo como instrumentos de comunicación en Europa”. Boletín de Marketing, núm. 28, pp. 9-14.

Laver, J. (2006). Breve historia del traje y la moda. Madrid: Cátedra.

Martín Cerro, R. (2010). El modelo de una agencia de comunicación de moda en España: Tal Cual Comunicación [trabajo fin de carrera]. Madrid: Universidad CEU San Pablo.

Martín Martín, F. (2012). Comunicación empresarial e institucional. Madrid: Universitas. Massot, D. (2008). Agatha Ruiz de la Prada. Navarra: EUNSA.

Palomo-Lovinski, N. (2011). Los diseñadores de moda más influyentes. Barcelona: Electa. Pastor, J. (2012). Creatividad e innovación. Madrid: EOI.

Ramírez, T. (1995). Gabinetes de comunicación. Barcelona: Bosch.

Real Academia Española.

_(2012a). Diccionario de la lengua española. Madrid: DRAE.

_(2012b). Diccionario panhispánico de dudas. Madrid: DRAE. 
Suárez Alba, A. (1991). "Las empresas que asesoran empresas”. En Fernández Collado, C. (coord.). La comunicación en organizaciones. México D. F.: Trillas.

Tal Cual Comunicación (2013). “Informe anual”. Madrid: Tal Cual Comunicación.

Universidad CEU San Pablo (2010). "Memoria Master de Comunicación en Moda y Belleza”. Madrid: Universidad CEU San Pablo. 